\title{
Hidden Sector Supergravity Breakdown
}

\author{
Hans Peter Nilles ${ }^{\text {a* }}$ \\ aPhysikalisches Institut, Universität Bonn, Nussallee 12, D-53115 Bonn, Germany \\ Gravity mediated supersymmetry breakdown originated from a study of gaugino condensation in a hidden \\ sector. We review this mechanism of supergravity breakdown from the original formulation in the early eighties \\ to its natural implementation in superstring theory and M-theory. In the latter case hidden and observable sector \\ become separated geometrically and supersymmetry is broken dynamically on a hidden wall.
}

\section{Introduction}

In the early 1980's considerations of the hierarchy problem in the standard model of strong and electroweak interactions (as well as grand unified theories) strengthened the interest in applications of supersymmetry to particle physics. I was at SLAC at that time and I remember being intrigued by the idea that the scale of dynamically broken supersymmetry [1] would explain the weak mass scale of the standard model and induce the breakdown of $S U(2) \times U(1)$. Still this goal was not so easy to achieve. After the first excitement of model building in the framework of global supersymmetry, problems started to appear. One of them was the problem of the cosmological constant that was strictly positive in spontaneously broken supersymmetry. In addition, the tree level supertrace formulae [2] made it difficult to consistently push up the superpartners to a high mass scale and there was no known sensible mechanism to generate gaugino masses. A new symmetry (Rsymmetry) was needed to assure proton stability, but the necessary breakdown of that symmetry led to problems with unacceptable axions. It soon became clear that realistic models had to be quite complicated and that supersymmetry breakdown should be somewhat remote from the standard model itself.

With this in mind I decided that the right thing

\footnotetext{
*Invited talk presented at the symposium "Thirty Years of Supersymmetry", University of Minnesota, October 2000. Work supported by grants of the European community RTN programmes HPNR-CT-2000-00131, 00148 and 00152 .
}

to do was to learn supergravity. After all gravity existed in nature and should be included sooner or later in the framework of the standard model. It was particularly imppressive for me that the local version of supersymmetry included gravity automatically (with the gravitino as gauge particle). In fall 1981 I was moving to CERN as a fellow and this was the right place to learn supergravity. The papers to read were [3,4] and two of the authors, Sergio Ferarra and Luciano Girardello were at CERN.

In this talk I would like to present the origin and status of hidden sector supergravity in three steps. The suggestion of the scheme [5] came in spring 1982 and led to hectic activities, so that in the beginning of 1983 the picture of what is today called "gravity mediation" was pretty complete. A second important step of the development came in 1985 after the "string revolution" initiated by Green and Schwarz [6]. Here the "hidden $E_{8^{-}}$ sector" of the heterotic string played a crucial role and the moduli fields of the $d=10$ dimensional string theory entered the stage. This will be discussed in chapter 3. More recently, with the advances of string dualities and the formulation of M-theory [7,8] some of the remaining questions could be clarified. The hidden sector now became a hidden wall and this version of the scheme will be presented in chapter 4 .

\section{1982: Hidden Sector Supergravity}

In a supersymmetric framework we wanted to understand the breakdown scale of $S U(2) \times U(1)$ 
(thus the $\mathrm{W}$ - and Z-mass) as a consequence of supersymmetry breakdown $\left(M_{\mathrm{S}}\right)$. The hierarchy problem was rephrased: why is $M_{\mathrm{S}}$ so small compared to the grand unified scale $M_{\mathrm{GUT}}$ or the Planck scale $M_{\text {Planck }}$ ? A satisfactory scheme would have been a dynamical of breakdown supersymmetry, such as fermion-antifermion condensation or the condensation of gauge fermion bilinears: gaugino condensation. Unfortunately, developments in 1981 led to the conclusion, that in the simplest model, globally supersymmetric pure $\mathrm{SU}(\mathrm{N})$ gauge theory, supersymmetry was not broken by the formation of gaugino condensates [9 11].

Meanwhile my study of supergravity made progress and I could handle some simple models. As an exercise I considered the effective Langrangian for pure supersymmetric YangMills theory as formulated by Veneziano and Yankielowicz [10] in its local supersymmetric version. Some modifications had to be made to finetune the cosmological constant. The result was surprising: supergravity was broken in the presence of a gaugino condensate [5]. Apart from the Polonyi superpotential 12] we had a second example of local supersymmetry breakdown with a vanishing cosmological constant.

But how could that be consistent with the apperent no-go theorem in the globally supersymmetric theory? Let us assume that $\Lambda$, the renormalization group invariant scale of the gauge interaction sets the scale for gaugino condensation, thus

$$
<\chi \chi>\approx \Lambda^{3}
$$

where $\chi$ denotes the gaugino. The supersymmetry breakdown scale $M_{\text {SUSY }}$ (as a vacuum expectation value of an auxiliary field) was here given by

$M_{\mathrm{SUSY}}^{2} \approx \frac{\Lambda^{3}}{M_{\mathrm{P}}}$

where $M_{\mathrm{P}}$ denotes the Planck scale.

Thus everything was consistent, in the global limit $M_{\mathrm{P}} \rightarrow \infty$ one obtains $M_{\mathrm{SUSY}} \rightarrow 0$ and unbroken supersymmetry. In spontaneously broken supergravity the gravitino becomes massive:

$m_{3 / 2} \approx \frac{\Lambda^{3}}{M_{\mathrm{P}}^{2}}$

This was very exciting since we had the possibility of dynamically broken supergravity in a "hidden sector" with an additional Planck mass suppression for the gravitino mass. What would that mean in case of coupling to the "observable sector" (the supersymmetric extension of the standard model). In the Polonyi case this had not been studied yet: in any case one expected (at that time!) $\operatorname{Str} M^{2}=0$ for the chiral multiplets and (in addition) no convincing way to generate gaugino masses (i.e. for the gauginos in the observable sector).

In the present case, however, there was a way to generate gaugino masses in the observable sector. Supergravity contains 4 -fermion interactions

$\frac{h(\phi)}{M_{\mathrm{P}}^{2}}(\chi \chi)(\chi \chi)$,

where $h(\phi)$ is a function of scalar fields $\phi$.

Imagine now a mixing term with one pair of observable sector gauginos (like the gluinos, winos, etc.) and a second pair of hidden sector gauginos with a nonvanishing vacuum expectation value. With this one obtains soft supersymmetry breaking gaugino masses in the observable sector and a completely satisfactory model [5]. Masses for squarks and sleptons appear via radiative corrections from the soft gaugino masses. The scale of soft supersymmetry breaking parameters is determined by the gravitino mass (with potential suppression factors). A detailed discussion can be found in ref. 13]. In this way the general picture of hidden sector supergravity breakdown (or gravity mediated supersymmetry breakdown) emerged. One needs two sectors:

- the observable sector including quarks, leptons, gauge particles and Higgs bosons as well as their supersymmetric partners and

- a hidden sector coupled to the observable sector through interactions of gravitational strength. 
The hidden sector provides supersymmetry breakdown at an intermediate scale and leads to a gravitino mass at a rather low scale. Soft breaking terms of order of the gravitino mass (or a few orders of magnitude smaller) appear in the observable sector and lead to a phenomenological satisfactory version of the supersymmetric extension of the standard model of strong and electroweak interactions.

Up to this point the supergravity action had only been constructed for one chiral multiplet. A more detailed analysis of the low energy effective action would require a generalization of this work. Such a generalization was subsequently presented by Cremmer, Ferrara, Girardello and van Proyen 14 stressing in particular that the supertrace formulae of global supersymmetry was no longer valid in this case. Chamseddine, Arnowit and Nath 15] quoted similar results in the framework of a grand unified theory, while Barbieri, Ferrara and Savoy [16] presented a supersymmetric extension of the standard model with soft scalar mass terms, exploiting the new $\operatorname{Str} M^{2}$ formulae in local supersymmetry. Srednicki, Wyler and myself 17 completed this analysis of soft SUSY breaking terms with among others the introduction of the A-parameters. The relevance of the gauge kinetic function $(f)$ for the generation of soft gaugino masses was pointed out in ref. [18]. A general comprehensive discussion was given in 19,20]. So within nine months the full picture had been completed. We now had a consistent scheme with (dynamically) broken supersymmetry and a vanishing cosmological constant but without tachyonic instabilities or harmful axions. Gaugino masses and A-parameters were generated naturally: a consequence of the breakdown of continuous Rsymmetry to discrete R-parity. Problems with flavour changing neutral currents (the so-called flavour problem) could be avoided by assuming certain degeneracies of soft scalar mass terms, which appeared automatically in case of gaugino masses as the dominant source of supersymmetry breakdown.

Still today "Hidden Sector Supergravity" appears as the most promising $\mathrm{d}=4$ set-up for a low energy effective theory that extends the standard model and avoids the hierarchy problem.
Remains the question of how this fits into "Grand Scenario", i.e. Strings and/or extra dimensions.

\section{1984: Towards Higher Dimensions and String Theory}

In their classic paper [6] on anomaly cancellation in $S 0(32)$ superstring theory, Green and Schwarz initiated a vast research effort in that field. Very soon new string constructions, such as the heterotic string theories 21,22] appeared and gave hope for an application in particle physics. Especially the heterotic $E_{8} \times E_{8}$ theory seemed very promising in this respect. Compactification of six dimensions on a Ricci flat manifold was shown to lead to supersymmetric models in $d=4$ dimensions that could serve as candidates for a supersymmetric extension of the standard model [23]. In its simplest form (the so-called standard embedding) this led to a theory with gauge group $E_{6} \times E_{8}$ and quarks and lepton superfields in the 27-dimensional representation to $E_{6}$. Thus all the observable fields were contained in the $E_{6}$ sector.

With Jean Pierre Derendinger and Luis Ibanez at CERN, we were excited about this fact. We had now a candidate theory which

(i) naturally contained an observable $\left(E_{6}\right)$ sector and a hidden $\left(E_{8}\right)$ sector, that were

(ii) connected only via interactions of gravitational strength, and

(ii) in the simplest model the hidden sector consisted of a pure supersymmetric gauge theory.

This was exactly the picture of hidden sector supergravity we have discussed in the previous section: supersymmetry is potentially broken by gaugino condensation in the hidden sector. It naturally appeared in the framework of superstring theories. Still, it was necessary to clarify the details. For this one needed a simple way to arrive at an at least qualitative description of the low energy effective action. Such a method was supplied in the scheme of reduction and truncation as defined in ref. 24] which we will describe 
shortly. With this machinery at hand we could explicitely discuss the question of supersymmetry breakdown in this set-up, as given in ref. [25]. Similar results were found independently in ref. [26]. What we see is that the messenger fields that are responsible for the mediation of supersymmetry breakdown are a combination of the string theory dilaton and moduli fields that describe the size and shape of the extra compactified dimensions. In the simple case considered here they are given by the moduli fields $S$ and $T$ that will be defined below. We shall be quite brief in our discussion here and refer the reader to ref. 27] for more details. For a more recent review see ref. 28].

\subsection{Supersymmetry breakdown in het- erotic string theory}

In our review of supersymmetry breakdown in the $d=10$ weakly coupled $E_{8} \times E_{8}$ theory we shall start from the $d=10$ effective field theory and go to $d=4$ dimensions via the method of reduction and truncation explained in ref. [24]. In string theory compactified on an orbifold this would describe the dynamics of the untwisted sector. We retain the usual moduli fields $S$ (the dilaton) and $T$ (the overal size of compactified extra dimensions) as well as matter fields $C_{i}$ that transform nontrivially under the observable sector gauge group. In this approximation, the Kähler potential is given by [24,27]

$G=-\log (S+\bar{S})-3 \log \left(T+\bar{T}-2 C_{i} \bar{C}_{i}\right)+\log |W|^{2}(5)$

with superpotential

$W(C)=d_{i j k} C_{i} C_{j} C_{k}$

and the gauge kinetic function is given by the dilaton field

$f=S$.

We assume the formation of a gaugino condensate $\langle\chi \chi\rangle=\Lambda^{3}$ in the hidden sector where $\Lambda$ is the renormalization group invariant scale of the confining hidden sector gauge group. The gaugino condensate appears in the expression for the auxiliary components of the chiral superfields 18

$F_{j}=\left(G^{-1}\right)_{j}^{k}\left(\exp (G / 2) G_{k}+\frac{1}{4} f_{k}(\chi \chi)\right)+\ldots$ which are order parameters for supersymmetry breakdown. Minimizing the scalar potential we find $F_{S}=0, F_{T} \neq 0$ and a vanishing cosmological constant. Supersymmetry is broken and the gravitino mass is given by [29]

$m_{3 / 2}=\frac{\left\langle F_{T}\right\rangle}{T+\bar{T}} \approx \frac{\Lambda^{3}}{M_{P}^{2}}$

and $\Lambda=10^{13} \mathrm{GeV}$ would lead to a gravitino mass in the $\mathrm{TeV}$ - range. A first inspection of the soft breaking terms in the observable sector gives a surprising result. They vanish in this approximation. Scalar masses are zero because of the no-scale structure [30] in (5) (coming from the fact that we have only included fields of modular weight -1 under $\mathrm{T}$-duality in this case [31]). In a more general situation we might expect scalar masses $m_{0}$ comparable to the gravitino mass $m_{3 / 2}$ and the above result $m_{0}=0$ would just be an artifact of the chosen approximation at the classical level. Thus soft scalar masses are strongly model dependent. Gaugino masses $m_{1 / 2}$ are easier to discuss. They are given by

$m_{1 / 2}=\frac{\frac{\partial f}{\partial S} F_{S}+\frac{\partial f}{\partial T} F_{T}}{2 \operatorname{Re} f}$

and with $f=S$ and $F_{S}=0$ we obtain $m_{1 / 2}=$ 0 . One loop corrections will change this picture as can be seen already by an inspection of the Green-Schwarz anomaly cancellation counter terms, as they modify $f$ at one loop. In the simple example of the so-called standard embedding with gauge group $E_{6} \times E_{8}$ we obtain [27,32]

$f_{6}=S+\epsilon T ; \quad f_{8}=S-\epsilon T$.

This dependence of $f$ on $T$ will via (10) lead to nonvanishing gaugino masses which, however, might be small compared to $m_{3 / 2}$ and $m_{0}$ since $\epsilon T$ is considered a small correction to the classical result. This might be problematic when applied to the class of supersymmetric extension of the standard model, where $m_{0}$ is large compared to $m_{1 / 2}$. With the large difference of the soft scalar and the gaugino masses a sizeable fine tuning is needed to induce the breakdown of electroweak symmetry at the correct scale 33]. The smallness of the gaugino masses might also lead to a problem in 
the context of relic abundances of the lightest superparticles (LSP) [34]. Ideally one would like to consider again a model where the main source of supersymmetry breaking is given by the gaugino masses.

\subsection{The role of the antisymmetric tensor field}

There are a few things that should be retained from this discussion that are not so transparent in the short presentation given above. In particular it is the role of the two index antisymmetric tensor field $B_{M N}$ that is important. In $d=10$ supergravity the (3 index) field strength $H_{M N P}$ is not just the curl of $B$ but has to be supplied with so-called Chern Simons terms [35].

We now want to stress the following points that will also be important in the discussion in the next section.

- The superpotential of the $d=4$ effective action originates in the co-called Yang-MillsChern-Simons term $\omega^{\mathrm{YM}}$.

- The Green Schwarz counterterms of anomaly cancellation, will provide (among others) a one-loop corection to the gauge kinetic function, that is classicaly just given by $S$

- In $d=4$ the real part of $S$ is given by $g^{-2}$, thus the gauge coupling constant $g$ is a field dependent quantity.

- In the $d=10$ dimensional theory the field strength $H$ and the gaugino bilinears $(\chi \chi)$ appear in a complete square, such that at the classical level the potential is positive definite.

- As a result of this a nonvanishing vacuum expectation value of $H$ will trigger supersymmetry breakdown and the dilaton will adjust its vacuum expectation value such that the cosmological constant vanishes.

- A vanishing value of $\langle H\rangle$ will then lead to a situation where $S$ runs to infinity with a vanishing gauge coupling constant.

\subsection{Open questions}

Certainly the potential runaway of the dilaton $S$ is an open problem in this scenario (as in any theory based on perturbative string theory ), and up to today no really satisfactory solution has been proposed. Other problems concern the exact form of the scalar potential. While at the classical level the vacuum energy vanishes, this is certainly not obvious in the quantum theory. As a result of this, it is quite difficult to obtain a statement about even the qualitative form of the potential in a model independent way. This, of course, implies that the soft supersymmetric breaking parameters (like squark and slepton masses as well as A-parameters) are strongly model dependent. In contrast, the situation for the gaugino masses is much simpler (as usual) and maybe they could even be dominant in a realistic framework. Their origin in the simple set-up is a direct consequence of the Green-Schwarz counterterms in the lowenergy effective action. In perturbative string theory they can be obtained through a one loop calculation [36] that agrees with the former result in the limit of large $T$.

In a next step, one would like to use more explicit input from string theory. One approach would be to exploit the presence of stringy symmetries [37]38], like $T$-duality, that should be valid in perturbative string theory. In fact, this picture of $T$-duality can be described successfully for the classical effective action [39 42]. There appear, however, problems when one incorporates loop effets, as now the $S$ and $T$-moduli mix. A successful implemenation of $T$-duality then seems to require an understanding of $S$-duality as well. $S$-duality, however, is not an explicit symmetry of the perturbative low energy effective action, and this is probably the reason, why this approach can not be completed 43 45]. The problem of the runaway dilaton, and thus the determination of the vacucum expextation value of $S$ seems to require a nonperturbative treatment and no really convincing scheme has emerged. As we have seen above this problem might be intimately related to the origin of a vacuum expectation value of the three-index tensor field strengh $\langle H\rangle$ and the apearence of Chern-simons terms. Still, a better understanding of string symmetries would be the 
road to a better control of the low energy effective action.

\section{1995: Towards $d=11$ and (Heterotic) M-theory}

A new step towards the understanding of nonperturbative dualities in string theory came in 1995 with the formulation of the concept of Mtheory [7,8 and its embedding in $d=11$ dimensional space time. For our purposes we are especially interested in the $E_{8} \times E_{8}$ version as constructed by Horava and Witten [46, 47]. The new aspect of this picture is the fact that now hidden and observable sector become separated geometrically. Thus hidden sector supergravity breakdown now becomes the breakdown of supergravity at a hidden brane, and again gaugino condensation is the preferred mechanism as suggested by Horava 48 and Olechowski, Yamaguchi and myself 49. Early related work has been done in 50,51

Let us now reconsider these questions in the strongly coupled $E_{8} \times E_{8}-M$-theory in somewhat more detail. The effective action is given by (for details see 477)

$$
\begin{gathered}
L=\frac{1}{\kappa^{2}} \int d^{11} x \sqrt{g}\left[-\frac{1}{2} R+\ldots\right]+\ldots \\
+\frac{1}{2 \pi\left(4 \pi \kappa^{2}\right)^{2 / 3}} \int d^{10} x \sqrt{g}\left[-\frac{1}{4} F_{A B}^{a} F^{a A B}+\ldots\right] .
\end{gathered}
$$

Compactifying to $d=4$ we obtain [53] (with the correction pointed out in ref. [52])

$$
\begin{aligned}
G_{N} & =8 \pi \kappa_{4}^{2}=\frac{\kappa^{2}}{8 \pi^{2} V \rho}, \\
\alpha_{G U T} & =\frac{\left(4 \pi \kappa^{2}\right)^{2 / 3}}{V}
\end{aligned}
$$

with $V=R^{6}$ and $\pi \rho=R_{11}$. Fitting $G_{N}$ and $\alpha_{G U T}=1 / 25$ then gives $R_{11} M_{11}$ of order 10 and $M_{11} R \approx 2.3$. The rather large value of the $d=4$ reduced Planck Mass $M_{P}=\kappa_{4}^{-1}$ is obtained as a result of the fact that $R_{11}$ is large compared to $R$.

We now perform a compactification using the method of reduction and truncation as above. For the metric we write 49,54

$g_{M N}=\left(\begin{array}{ccc}e^{-\gamma} e^{-2 \sigma} g_{\mu \nu} & & \\ & e^{\sigma} \delta_{m n} & \\ & & e^{2 \gamma} e^{-2 \sigma}\end{array}\right)$

with $M, N=1 \ldots 11 ; \mu, \nu=1 \ldots 4 ; m, n=$ $5 \ldots 10 ; 2 R_{11}=2 \pi \rho=M_{11}^{-1} e^{\gamma} e^{-\sigma}$ and $V=$ $e^{3 \sigma} M_{11}^{6}$. At the classical level this leads to a Kähler potential as in (5)

$K=-\log (\mathcal{S}+\overline{\mathcal{S}})-3 \log \left(\mathcal{T}+\overline{\mathcal{T}}-2 C_{i} \bar{C}_{i}\right)$

with

$$
\begin{aligned}
\mathcal{S} & =\frac{2}{(4 \pi)^{2 / 3}}\left(e^{3 \sigma} \pm i 24 \sqrt{2} D\right), \\
\mathcal{T} & =\frac{\pi^{2}}{(4 \pi)^{4 / 3}}\left(e^{\gamma} \pm i 6 \sqrt{2} C_{11}\right)
\end{aligned}
$$

where $D$ and $C_{11}$ fields are defined by

$$
\begin{aligned}
\frac{1}{4 !} e^{6 \sigma} G_{11 \lambda \mu \nu} & =\epsilon_{\lambda \mu \nu \rho}\left(\partial^{\rho} D\right), \\
C_{11 i \bar{j}} & =C_{11} \delta_{i \bar{j}}
\end{aligned}
$$

and $x^{i}\left(x^{\bar{j}}\right)$ is the holomorphic (antiholomorphic) coordinate of the Calabi-Yau manifold. The imaginary part of $\mathcal{S}(\operatorname{Im} \mathcal{S})$ corresponds to the model independent axion, and the gauge kinetic function is $f=\mathcal{S}$. This is very similar to the weakly coupled case. Before drawing any conclusion from these formulae, however, we have to discuss a possible obstruction at the one loop level. It can be understood from the mechanism of anomaly cancellation [53]. For the 3-index tensor field $H$ in $d=10$ supergravity to be well defined one has to satisfy $d H=\operatorname{tr} F_{1}^{2}+\operatorname{tr} F_{2}^{2}-\operatorname{tr} R^{2}=0$ cohomologically. In the simplest case of the standard embedding one assumes $\operatorname{tr} F_{1}^{2}=\operatorname{tr} R^{2}$ locally and the gauge group is broken to $E_{6} \times E_{8}$. Since in the M-theory case the two different gauge groups live on the two different boundaries of space-time such a cancellation point by point is no longer possible. We expect nontrivial vacuum expectation values (vevs) of

$(d G) \propto \sum_{i} \delta\left(x^{11}-x_{i}^{11}\right)\left(\operatorname{tr} F_{i}^{2}-\frac{1}{2} \operatorname{tr} R^{2}\right)$

at least on one boundary $\left(x_{i}^{11}\right.$ is the position of $i$-th boundary). In the case of the standard embedding we would have $\operatorname{tr} F_{1}^{2}-\frac{1}{2} \operatorname{tr} R^{2}=\frac{1}{2} \operatorname{tr} R^{2}$ 
on one and $\operatorname{tr} F_{2}^{2}-\frac{1}{2} \operatorname{tr} R^{2}=-\frac{1}{2} \operatorname{tr} R^{2}$ on the other boundary. This might pose a severe problem since a nontrivial vev of $G$ might be in conflict with supersymmetry $\left(G_{11 A B C}=H_{A B C}\right)$. The supersymmetry transformation law in $d=11$ reads

$\delta \psi_{M}=D_{M} \eta+\frac{\sqrt{2}}{288} G_{I J K L}\left(\Gamma_{M}^{I J K L}+\ldots\right) \eta+\ldots$

Supersymmetry will be broken unless e.g. the derivative term $D_{M} \eta$ compensates the nontrivial vev of $G$. Witten has shown 53 that such a cancellation can occur and constructed the solution in the linearized approximation (linear in the expansion parameter $\kappa^{2 / 3}$ ) which corresponds to the large $T$-limit in the weakly coupled theory

The supersymmetric solution can be shown to lead [49] to a nontrivial dependence of the $\sigma$ and $\gamma$ fields with respect to $x^{11}$ :

$\frac{\partial \gamma}{\partial x^{11}}=-\frac{\partial \sigma}{\partial x^{11}}=\frac{\sqrt{2}}{24} \frac{\int d^{6} x \sqrt{g} \omega^{A B} \omega^{C D} G_{A B C D}}{\int d^{6} x \sqrt{g}}$

where the integrals are over the Calabi-Yau manifold and $\omega$ is the corresponding Kähler form. Formula (23) contains all the information to deduce the effective $d=4$ supergravity theory, including the Kähler potential and the gauge kinetic function [49,54. A definition of our $\mathcal{S}$ and $\mathcal{T}$ fields in the four-dimensional theory now requires an average over the 11-dimensional interval. We therefore write

$$
\begin{aligned}
\mathcal{S} & =\frac{2}{(4 \pi)^{2 / 3}}\left(e^{3 \bar{\sigma}} \pm i 24 \sqrt{2} \bar{D}\right), \\
\mathcal{T} & =\frac{\pi^{2}}{(4 \pi)^{4 / 3}}\left(e^{\bar{\gamma}} \pm i 6 \sqrt{2} \bar{C}_{11}\right)
\end{aligned}
$$

where bars denote averaging over the 11th dimension. It might be of some interest to note that the combination $\mathcal{S T}^{3}$ is independent of $x^{11}$ even before this averaging procedure took place.

$\exp (3 \sigma)$ represents the volume of the sixdimensional compact space in units of $M_{11}^{-6}$. The $x^{11}$ dependence of $\sigma$ then leads to the geometrical picture that the volume of this space varies with $x^{11}$ and differs at the two boundaries. In the given approximation, this variation is linear, and

${ }^{\dagger}$ For a discussion beyond this approximation in the weakly coupled case see refs. [55,56]. for growing $R_{11}$ the volume on the $E_{8}$ side becomes smaller and smaller. At a critical value of $R_{11}$ the volume will thus vanish and this will provide us with an upper limit on $R_{11}$. For the phenomenological applications we then have to check whether our preferred choice of $R_{11}$ that fits the correct value of the $d=4$ Planck mass satisfies this bound. Although the coefficients are model dependent we find in general that the bound can be satisfied, but that $R_{11}$ is quite close to its critical value. A choice of $R_{11}$ much larger than (few $\left.\times 10^{15} \mathrm{GeV}\right)^{-1}$ is therefore not permitted.

This variation of the volume is the analogue of the one loop correction of the gauge kinetic function (11) in the weakly coupled case and has the same origin, namely a Green-Schwarz anomaly cancellation counterterm. In fact, also in the strongly coupled case we find [49], as a consequence of (23), corrections for the gauge coupling constants at the $E_{6}$ and $E_{8}$ side.

Gauge couplings will no longer be given by the (averaged) $\mathcal{S}$-field, but by that combination of the (averaged) $\mathcal{S}$ and $\mathcal{T}$ fields which corresponds to the $\mathcal{S}$-field before averaging at the given boundary:

$f_{6,8}=\mathcal{S} \pm \alpha \mathcal{T}$

at the $E_{6}\left(E_{8}\right)$ side respectively 3 . The critical value of $R_{11}$ will correspond to infinitely strong coupling at the $E_{8}$ side $\mathcal{S}-\alpha \mathcal{T}=0$ (Notice the similarity to (11) in the weakly coupled limit). Since we are here close to criticality a correct phenomenological fit of $\alpha_{\mathrm{GUT}}=1 / 25$ should include this correction $\alpha_{\text {GUT }}^{-1}=\mathcal{S}+\alpha \mathcal{T}$ where $\mathcal{S}$ and $\alpha \mathcal{T}$ give comparable contributions. This is a difference to the weakly coupled case, where in $f=S+\epsilon T$ the latter contribution was small compared to $S$. Observe that this picture of a loop correction $\alpha \mathcal{T}$ to be comparable to the tree level result still makes sense in the perturbative expansion, since $f$ does not receive further perturbative corrections beyond one loop [37.

\footnotetext{
${ }^{\ddagger}$ With $V$ depending on $x^{11}$ we have to specify which values should be used in eq. (14). The appropriate choice in the expression for $G_{N}$ is the average value of $V$ while in the expression for $\alpha_{G U T}$ we have to use $V$ evaluated at the $E_{6}$ border.

${ }^{\S}$ With the normalization of the $\mathcal{T}$ field as in (25), $\alpha$ is a quantity of order unity.
} 


\subsection{Supersymmetry breakdown in M- theory}

In a next step we are now ready to discuss the dynamical breakdown of supersymmetry via gaugino condensation in the strongly coupled Mtheory picture. In analogy to the previous discussion we start investigating supersymmetry transformation laws in the higher-dimensional (now $d=11$ ) field theory [48]:

$$
\begin{aligned}
\delta \psi_{A} & \sim D_{A} \eta+G_{I J K L}\left(\Gamma_{A}^{I J K L}-8 \delta_{A}^{I} \Gamma^{J K L}\right) \eta \\
& -\delta\left(x^{11}\right)\left(\bar{\chi}^{a} \Gamma_{B C D} \chi^{a}\right) \Gamma_{A}^{B C D} \eta+\ldots(27) \\
\delta \psi_{11} & \sim D_{11} \eta+G_{I J K L}\left(\Gamma_{11}^{I J K L}-8 \delta_{11}^{I} \Gamma^{J K L}\right) \eta \\
& +\delta\left(x^{11}\right)\left(\bar{\chi}^{a} \Gamma_{A B C} \chi^{a}\right) \Gamma^{A B C} \eta+\ldots
\end{aligned}
$$

where gaugino bilinears appear in the right hand side of both expressions. It can therefore be expected that gaugino condensation breaks supersymmetry. Still the details have to be worked out. In the $d=10$ example, the gaugino condensate and the three-index tensor field $H$ contributed to the scalar potential in a full square. This has led to a vanishing cosmological constant as well as the fact that $F_{S}=0$ at the classical level. Hořava has observed 48] that a similar mechanism might be in operation in the $d=11$ theory After a careful calculation this leads to a vanishing variation $\delta \psi_{A}=0$. In our model (based on reduction and truncation) we can now compute these quantities explicitly. We assume gaugino condensation to occur at the $E_{8}$ boundary

$$
\left\langle\bar{\chi}^{a} \Gamma_{i j k} \chi^{a}\right\rangle=\Lambda^{3} \epsilon_{i j k}
$$

where $\Lambda<M_{\mathrm{GUT}}$ and $\epsilon_{i j k}$ is the covariantly constant holomorphic 3 -form. This leads to a nontrivial vev of $G_{11 A B C}$ at this boundary and supersymmetry is broken 1 . At that boundary we obtain $F_{\mathcal{S}}=0$ and $F_{\mathcal{T}} \neq 0$ as expected from the fact that the component $\psi_{11}$ of the 11-dimensional gravitino plays the role of the goldstino.

\footnotetext{
厅 One might speculate that a nontrivial vev of $D_{A} \eta$ might be operative here as in the case without gaugino condensation (see discussion after eq. (22)). However, the special values of $H_{i j k} \propto \epsilon_{i j k}$ necessary to cancel the contribution of the gaugino condensate do not permit such a mechanism (see footnote 6 in ref. [53]).
}

In the effective $d=4$ theory we now have to average over the 11th dimension leading to

$$
\left\langle F_{\mathcal{T}}\right\rangle \approx \frac{1}{2} \mathcal{T} \frac{\int d x^{11} \delta \psi_{11}}{\int d x^{11}}
$$

as the source of SUSY breakdown.

This will then allow us to compute the size of supersymmetry breakdown on the observable $E_{6}$ side. Gravitational interactions play the role of messengers that communicate between the two boundaries. This effect can be seen from (14): large $R_{11}$ corresponds to large $M_{P}$ and $\left\langle F_{\mathcal{T}}\right\rangle$ gives the effective size of SUSY breaking on the $E_{6}$ side $\left(R_{11} \rightarrow \infty\right.$ implies $\left.M_{P} \rightarrow \infty\right)$. The gravitino mass is given by

$m_{3 / 2}=\frac{\left\langle F_{\mathcal{T}}\right\rangle}{\mathcal{T}+\overline{\mathcal{T}}} \approx \frac{\Lambda^{3}}{M_{P}^{2}}$

(similar to (9) in the weakly coupled case) and we expect this to represent the scale of soft supersymmetry breaking parameters in the observable sector [33]. These soft masses are determined by the coupling of the corresponding fields to the goldstino multiplet. As we have seen before, we cannot compute the scalar masses reliably. In the naive approximation used here we obtain $m_{0}=0$ because of the no-scale structure [30]. This might be an artifact of the approximation. Fields of different modular weight will receive contribution to $m_{0}$ of order $m_{3 / 2}$. For the mass of a field $C$ we have [31]

$m_{0}^{2}=m_{3 / 2}^{2}-F^{i} \bar{F}^{\bar{j}} \frac{Z_{i \bar{j}}-Z_{i} Z^{-1} \bar{Z}_{\bar{j}}}{Z}$

where $i, j=\mathcal{S}, \mathcal{T}$ and $Z$ is the moduli dependent coefficient of $C \bar{C}$ term appearing in the Kähler potential. Scalars of modular weight -1 will become massive through radiative corrections. This then leads to the expectation that $m_{3 / 2}$ should be in the TeV-region and $\Lambda \approx 10^{13} \mathrm{GeV}$. So far this is all similar to the weakly coupled case.

\subsection{Gaugino masses and WIMPs}

An important difference appears, however, when we turn to the discussion of observable sector gaugino masses (10). In the weakly coupled

"In realistic models $E_{8}$ is broken and $\Lambda$ is adjusted by model building. 
case they were zero at tree level and appeared only because of the radiative corrections at one loop (11). As a result of this small correction, gaugino masses were expected to be much smaller than $m_{3 / 2}$. In the strongly coupled case the ana$\log$ of $(10)$ is still valid

$m_{1 / 2}=\frac{\frac{\partial f_{6}}{\partial \mathcal{S}} F_{\mathcal{S}}+\frac{\partial f_{6}}{\partial \mathcal{T}} F_{\mathcal{T}}}{2 \operatorname{Re} f_{6}}$

and the 1-loop effect is encoded in the variation of the $\sigma$ and $\gamma$ fields from one boundary to the other. Here, however, the loop corrections are sizable compared to the classical result because of the fact that $R_{11}$ is close to its critical value. As a result we expect observable gaugino masses of the order of the gravitino mass. The problem of the small gaugino masses does therefore not occur in this situation. Independent of the question whether $F_{\mathcal{S}}$ or $F_{\mathcal{T}}$ are the dominant sources of supersymmetry breakdown, the gauginos will be heavy of the order of the gravitino mass. Thus it could very well be that gaugino masses are the dominant sources of supersymmetry breakdown in the observable sector. The exact relation between the soft breaking parameters $m_{0}$ and $m_{1 / 2}$ will be a question of model building. If in some models $m_{0} \ll m_{1 / 2}$ this might give a solution to the flavor problem. The no-scale structure found above might be a reason for such a suppression of $m_{0}$. As we have discussed above, this structure, however, is an artifact of our simplified approximation and does not necessarily survive in perturbation theory. At best it could be kept exact (but only for the fields with modular weight -1 ) in the $R_{11} \rightarrow \infty$ limit. The upper bound on $R_{11}$ precludes such a situation. With observable gaugino masses of order $m_{3 / 2}$ we also see that $m_{3 / 2}$ cannot be arbitrarily large and should stay in the $\mathrm{TeV}$ - range [49,50].

The question of the soft gaugino masses is, of course, very important for the determination of the mass of the lightest supersymmetric particle (LSP). This might be stable and provide a candidate for the dark matter found in the universe (a so-called WIMP, for weakly interacting massive particle). Given the present situation of the supersymmetric extension of the standard model, the LSP is most likely a gaugino of the wino- or bino-type. The relic abundance of these neutralinos in the early universe depends crucially on its mass and its coupling to ordinary matter. The mass is predominantly determined through the soft gaugino masses, while the interactions are mediated by the scalar partners of leptons. Too high a mass of the scalar partners leads then to a weak coupling of the LSP to ordinary matter, reducing the annihilation of WIMPs in the early universe; with the potential danger of an overcritical WIMP density. For this relic density of WIMPs to be both acceptable and cosmologically interesting, only certain ranges of soft scalar masses and gaugino masses are allowed. It turns out, that the heterotic M-theory could lead to such an acceptable scenario (in contrast to the the situation in the weakly coupled case). A detailed discussion can be found in ref. [57].

\subsection{The Super-Higgs mechanism}

In the previous sections the estimate of the gravitino mass was obtained 440 using a the simplified approximation according to which the higher dimensional bulk fields were integrated out via an averaging proceedure*. In this picture, the goldstino mode was represented by the lowest Kaluza-Klein $\Psi_{0}$ mode of a higher dimensional field $\Psi$. In the super-Higgs mechanism this mode supplies the additional degrees of freedom to render the gravitino massive. Qualitatively this simplified approximation does give a consistent picture, but there remain some open questions and potential problems when one looks into details of the super-Higgs mechanism. In this section we would like to point out these potential problems and show how they can be resolved. For a detailed explanation we refer the reader to [59]. We shall concentrate here on a qualitative discussion of the mechanism involved. This will include a discussion of the possible nature of the goldstino (is it a bulk or a wall field), the relation to the Scherk-Schwarz mechanism 60] in that context 61] and an upper limit for the gravitino mass in the present picture. We shall argue that a meaningful realization of the super-Higgs mechanism seems to require some modes in the bulk other

**A corresponding analysis in global supersymmetry has been performed in ref. 58 . 
than the graviton and the gravitino. Finally we shall comment on the phenomenological consequences of this findings, including a discussion of the nature of the soft breaking terms on both walls.

Specifically we want to address the following two questions:

(i) the nature of the massless gravitino in the presence of several $F$-terms闭 on different walls that cancel and lead to unbroken supersymmetry,

(ii) the identification of the goldstino in the case of broken supersymmetry.

The first question (i) arises because of a particalur nonlocal effect of supersymmetry breakdown first observed by Horava 48]. A given source of supersymmetry breakdown (parametrized by a vacuum expectation value (vev) of an auxiliary field $F$ ) on one wall could be compensated by a similar but opposite value $(-F)$ on another (separated) wall. Any calculation and approximation of the system thus has to reproduce this behaviour. The previously mentioned averaging proceedure over the bulk distance does this in a trivial way, leading to unbroken supersymmetry as expected. A detailed inspection of the gravitino, however, reveals a problem. If we start with the situation $F=0$ it is easy to define the massless gravitino $\Psi_{0}$ in the $d=4$ theory. Switching on a nontrivial $F$ on one brane and $(-F)$ on the other still should give a massless gravitino, but $\Psi_{0}$ turns out to be no longer a mass eigenstate. The resolution of this problem and the correct identification of the gravitino can be found in [59]. It is a particular combination of the possible gravitini that appear when one, for example, reduces a 5 -dimensional theory to a theory in $d=4$ on a finite $d=5$ interval. The theory on a $d=5$ circle would lead to $N=2$ supersymmetry in $d=4$ and two massless gravitini (zero modes on the circle). The $Z_{2}$ projection on the interval removes one of the gravitini and is $N=1$

$\dagger^{\dagger}$ We generically use the notation $F$-term for the source of supersymmetry breakdown. Depending on the specific situation this could represent a $D$-term or a gaugino condensate as well. supersymmetric. A nonvanishing vev of $F$ now interferes with the boundary conditions and the massless gravitino will be a linear combination of the zero mode and all the excited Kaluza-Klein modes whose coefficients will depend on $F$ (assuming, of course, unbroken supersymmetry due to a compensating vev $-F$ on another wall).

The second question (ii) deals with the nature of the goldstino (i.e. the longitudinal components of the gravitino) in the case of broken supersymmetry. Remember that the simplified averaging proceedure leads to a goldstino that corresponds to the lowest Kaluza-Klein mode $\Psi_{0}$ of a higherdimensional bulk field $\Psi$. Inspecting the gravitino mass matrix in this case reveals the fact that this field $\Psi_{0}$ is not a mass eigenstate, but mixes with infinitely many higher Kaluza-Klein modes $\Psi_{n}$. A consistent manifestation of a super-Higgs mechanism would require a diagonalization of this mass matrix and an identification of the goldstino. This problem has been solved in [59], by a suitable redefinition of the Kaluza-Klein modes. Thus it is shown that a consistent Super-Higgs mechanism is at work.

\section{The nature of hidden wall supersymme- try breakdown}

The resolution of these puzzles clarifies some of the other questions of the approach. It also allows us to generalize this to string theories of type I with D-branes and supersymmetry breakdown on distant branes. The qualitative picture, of course, is very similar.

- The nonlocality of the breakdown shows some resemblance to the breakdown of supersymmetry via the Scherk-Schwarz 60 mechanism. Here, however, the real goldstino of the spontaneous breakdown of supersymmetry can be unambiguously identified.

- The possibility to cancel the supersymmetry breakdown on a distant wall by a vev on the local wall tells us, that the mass splittings of broken supersymmetry have to be of order of the gravitino mass $m_{3 / 2}$ on both walls. 
- In terms of the physical quantities there is no real extra suppression, once we separate the walls by a large distance $R$. In the limit $R \rightarrow \infty$ we will have $M_{\text {Planck }} \rightarrow \infty$ as well. The suppression of the soft breaking parameters will always be gravitational.

- In general, when we have a system of many separated branes with potential sources of supersymmetry breakdown, the actual breakdown will be obtained by the sum of these contributions. The averaging proceedure will be very useful to decide whether supersymmetry is broken or not. The identification of the goldstino, however, is more difficult and requires a careful calculation.

- A successful implementation of the superHiggs mechanism will require some fields other than gravitino and graviton in the bulk周. This implies that in the absence of such fields a consistent spontaneous breakdown of supergravity might not be achieved.

We thus have a consistent scenario of supersymmetry breakdown in the framework of heterotic M-theory. It provides us with explicit formulae of soft supersymmetry breaking terms in the low energy effective theory. Interestingly enough these formulae differ from those obtained in the weakly coupled heterotic $E_{8} \times E_{8}$ theory, most notably with respect to the size of soft gaugino masses. The phenomenological properties of the scenario, therefore have to be reconsidered in various circumstances. This might include a discussion of the mass and nature of the LSP, the question of the universality of the soft scalar masses as well as possible new solutions to the strong $\mathrm{CP}$ problem. More work needs to be done to work out these questions in detail. In summary we can say that the picture of supersymmetry breakdown in the M-theoretic limit looks very promising. It is very similar to the weakly coupled case, but avoids the problem of the small gaugino masses. This has important consequences for the phenomenological and

\footnotetext{
$\ddagger \ddagger$ Usually they arise as modes of the higher dimensional supergravity multiplet.
}

cosmological properties of the effective models in four dimensions.

\section{Outlook}

Since its original formulation in 1982, gravity mediation appeared as a very attractive scheme to introduce spontaneous (dynamical) supersymmetry breakdown into the supersymmetric extension of the $S U(3) \times S U(2) \times U(1)$ standard model of strong, weak and electromagnetic interactions. Its natural implementation in (heterotic) string theory and M-theory stengthens the confidence in this mechanism of hidden sector supergravity breakdown. It gives us useful hints about the possibility of a local cancellation of the classical vacuum energy, the role of anomaly cancellation via the Green-Schwarz counterterms, the importance of underlying string symmetries and the specific role of the antisymmetric tensor fields in stringand M-theory.

Still there remain some open questions about the possible incorporation of $T$ - and $S$-duality, the microscopic origin of the vacucum expectation value of the $\langle H\rangle$ and $\langle G\rangle$ fields and their potential to avoid the run-away problem of the dilaton. The control of the cosmological constant beyond the classical level remains a problem and this makes it difficult to determine the soft scalar mass terms in a model independent way. Soft gaugino masses are easier to handle since their size seems to be controlled by the mechanism of anomaly cancellation. The most attractive scheme emerges in the case where gaugino masses are the dominant source of supersymmetry breakdown.

\section{REFERENCES}

1. E. Witten, "Dynamical Breaking Of Supersymmetry," Nucl. Phys. B 188, 513 (1981).

2. S. Ferrara, L. Girardello and F. Palumbo, "A General Mass Formula In Broken Supersymmetry," Phys. Rev. D 20, 403 (1979).

3. E. Cremmer, B. Julia, J. Scherk, P. van Nieuwenhuizen, S. Ferrara and L. Girardello, "Superhiggs Effect In Supergravity With 
General Scalar Interactions," Phys. Lett. B 79, 231 (1978).

4. E. Cremmer, B. Julia, J. Scherk, S. Ferrara, L. Girardello and P. van Nieuwenhuizen, "Spontaneous Symmetry Breaking And Higgs Effect In Supergravity Without Cosmological Constant," Nucl. Phys. B 147, 105 (1979).

5. H. P. Nilles, "Dynamically Broken Supergravity And The Hierarchy Problem," Phys. Lett. B 115, 193 (1982).

6. M. B. Green and J. H. Schwarz, "Anomaly Cancellation In Supersymmetric D=10 Gauge Theory And Superstring Theory," Phys. Lett. B 149, 117 (1984).

7. E. Witten, "String theory dynamics in various dimensions," Nucl. Phys. B 443, 85 (1995) hep-th/9503124.

8. C. M. Hull and P. K. Townsend, "Unity of superstring dualities," Nucl. Phys. B 438, 109 (1995) [hep-th/9410167].

9. E. Witten, "Constraints On Supersymmetry Breaking," Nucl. Phys. B 202, 253 (1982).

10. G. Veneziano and S. Yankielowicz, "An Effective Lagrangian For The Pure N=1 Supersymmetric Yang-Mills Theory," Phys. Lett. B 113, 231 (1982).

11. H. P. Nilles, "Is Supersymmetry Afraid Of Condensates?," Phys. Lett. B 112, 455 (1982).

12. J. Polonyi, "Generalization Of The Massive Scalar Multiplet Coupling To The Supergravity," Hungary Central Inst Res - KFKI-77-93 (77,REC.JUL 78) $5 p$.

13. H. P. Nilles, "Supergravity Generates Hierarchies," Nucl. Phys. B 217, 366 (1983).

14. E. Cremmer, S. Ferrara, L. Girardello and A. Van Proeyen, "Coupling Supersymmetric Yang-Mills Theories To Supergravity," Phys. Lett. B 116, 231 (1982).

15. A. H. Chamseddine, R. Arnowitt and P. Nath, "Locally Supersymmetric Grand Unification," Phys. Rev. Lett. 49, 970 (1982).

16. R. Barbieri, S. Ferrara and C. A. Savoy, "Gauge Models With Spontaneously Broken Local Supersymmetry," Phys. Lett. B 119, 343 (1982).

17. H. P. Nilles, M. Srednicki and D. Wyler, "Weak Interaction Breakdown Induced By
Supergravity," Phys. Lett. B 120, 346 (1983).

18. S. Ferrara, L. Girardello and H. P. Nilles, "Breakdown Of Local Supersymmetry Through Gauge Fermion Condensates," Phys. Lett. B 125, 457 (1983).

19. L. Hall, J. Lykken and S. Weinberg, "Supergravity As The Messenger Of Supersymmetry Breaking," Phys. Rev. D 27 (1983) 2359.

20. S. K. Soni and H. A. Weldon, "Analysis Of The Supersymmetry Breaking Induced By N=1 Supergravity Theories," Phys. Lett. B 126, 215 (1983).

21. D. J. Gross, J. A. Harvey, E. Martinec and R. Rohm, "Heterotic String Theory. 1. The Free Heterotic String," Nucl. Phys. B 256, 253 (1985).

22. D. J. Gross, J. A. Harvey, E. Martinec and R. Rohm, "Heterotic String Theory. 2. The Interacting Heterotic String," Nucl. Phys. B 267 (1986) 75.

23. P. Candelas, G. T. Horowitz, A. Strominger and E. Witten, "Vacuum Configurations For Superstrings," Nucl. Phys. B 258, 46 (1985).

24. E. Witten, "Dimensional Reduction Of Superstring Models," Phys. Lett. B 155, 151 (1985).

25. J. P. Derendinger, L. E. Ibanez and H. P. Nilles, "On The Low-Energy $\mathrm{D}=4$, $\mathrm{N}=1$ Supergravity Theory Extracted From The $\mathrm{D}=10, \mathrm{~N}=1$ Superstring," Phys. Lett. B 155, 65 (1985).

26. M. Dine, R. Rohm, N. Seiberg and E. Witten, "Gluino Condensation In Superstring Models," Phys. Lett. B 156, 55 (1985).

27. J. P. Derendinger, L. E. Ibanez and H. P. Nilles, "On The Low-Energy Limit Of Superstring Theories," Nucl. Phys. B 267, 365 (1986).

28. H. P. Nilles, "TASI lectures 1997: On the low-energy limit of string and M-theory," hepph/0004064.

29. H. P. Nilles, "Gaugino Condensation And Supersymmetry Breakdown," Int. J. Mod. Phys. A 5, 4199 (1990).

30. J. Ellis, C. Kounnas and D. V. Nanopoulos, "Phenomenological SU(1,1) Supergravity," Nucl. Phys. B 241, 406 (1984).

31. A. Brignole, L. E. Ibanez and C. Munoz, "To- 
wards a theory of soft terms for the supersymmetric Standard Model," Nucl. Phys. B 422, 125 (1994) hep-ph/9308271.

32. L. E. Ibanez and H. P. Nilles, "Low-Energy Remnants Of Superstring Anomaly Cancellation Terms," Phys. Lett. B 169, 354 (1986).

33. H. P. Nilles, "Supersymmetry, Supergravity And Particle Physics," Phys. Rept. 110 (198

34. G. Jungman, M. Kamionkowski and K. Griest, "Supersymmetric dark matter," Phys. Rept. 267, 195 (1996) hep-ph/9506380.

35. A. H. Chamseddine, "Interacting Supergravity In Ten-Dimensions: The Role Of The Six - Index Gauge Field," Phys. Rev. D 24, 3065 (1981).

36. L. J. Dixon, V. Kaplunovsky and J. Louis, "Moduli dependence of string loop corrections to gauge coupling constants," Nucl. Phys. B 355, 649 (1991).

37. H. P. Nilles, "The Role Of Classical Symmetries In The Low-Energy Limit Of Superstring Theories," Phys. Lett. B 180, 240 (1986).

38. J. Lauer, J. Mas and H. P. Nilles, "Duality And The Role Of Nonperturbative Effects On The World Sheet," Phys. Lett. B 226, 251 (1989).

39. A. Font, L. E. Ibanez, D. Lust and F. Quevedo, "Supersymmetry Breaking From Duality Invariant Gaugino Condensation," Phys. Lett. B 245, 401 (1990).

40. H. P. Nilles and M. Olechowski, "Gaugino Condensation And Duality Invariance," Phys. Lett. B 248, 268 (1990).

41. S. Ferrara, N. Magnoli, T. R. Taylor and G. Veneziano, Phys. Lett. B 245, 409 (1990).

42. P. Binetruy and M. K. Gaillard, Phys. Lett. B 253, 119 (1991).

43. Z. Lalak, A. Niemeyer and H. P. Nilles, "Gaugino condensation, S duality and supersymmetry breaking in supergravity models," Nucl. Phys. B 453, 100 (1995) hepth/9503170.

44. Z. Lalak, A. Niemeyer and H. P. Nilles, "S dual Gaugino condensation and supersymmetry breaking," Phys. Lett. B 349, 99 (1995) hep-th/9410205.

45. Z. Lalak, S. Lavignac and H. P. Nilles, "String dualities in the presence of anomalous $\mathrm{U}(1)$ symmetries," Nucl. Phys. B 559, 48 (1999) hep-th/9903160.

46. P. Horava and E. Witten, "Heterotic and type I string dynamics from eleven dimensions," Nucl. Phys. B 460, 506 (1996) hepth/9510209.

47. P. Horava and E. Witten, "ElevenDimensional Supergravity on a Manifold with Boundary," Nucl. Phys. B 475, 94 (1996) hep-th/9603142].

48. P. Horava, "Gluino condensation in strongly coupled heterotic string theory," Phys. Rev. D 54, 7561 (1996) hep-th/9608019.

49. H. P. Nilles, M. Olechowski and M. Yamaguchi, "Supersymmetry breaking and soft terms in M-theory," Phys. Lett. B 415, 24 (1997) hep-th/9707143.

50. Z. Lalak and S. Thomas, "Gaugino condensation, moduli potential and supersymmetry breaking in M-theory models," Nucl. Phys. B 515, 55 (1998) hep-th/9707223.

51. A. Lukas, B. A. Ovrut and D. Waldram, "On the four-dimensional effective action of strongly coupled heterotic string theory," Nucl. Phys. B 532, 43 (1998) hepth/9710208.

52. J. O. Conrad, "Brane tensions and coupling constants from within M-theory," Phys. Lett. B 421, 119 (1998) hep-th/9708031.

53. E. Witten, Nucl. Phys. B 471, 135 (1996) hep-th/9602070.

54. H. P. Nilles, M. Olechowski and M. Yamaguchi, "Supersymmetry breakdown at a hidden wall," Nucl. Phys. B 530, 43 (1998) hepth/9801030.

55. H. P. Nilles and S. Stieberger, "String unification, universal one-loop corrections and strongly coupled heterotic string theory," Nucl. Phys. B 499, 3 (1997) hepth/9702110.

56 . S. Stieberger, " $(0,2)$ heterotic gauge couplings and their M-theory origin," Nucl. Phys. B 541, 109 (1999) hep-th/9807124.

57. Y. Kawamura, H. P. Nilles, M. Olechowski and M. Yamaguchi, "Relic abundance of neutralinos in heterotic string theory: Weak coupling vs. strong coupling," JHEP9806, 008 (1998) hep-ph/9805397. 
58. E. A. Mirabelli and M. E. Peskin, "Transmission of supersymmetry breaking from a 4-dimensional boundary," Phys. Rev. D 58, 065002 (1998) hep-th/9712214.

59. K. A. Meissner, H. P. Nilles and M. Olechowski, "Supersymmetry breakdown at distant branes: The super-Higgs mechanism," Nucl. Phys. B 561, 30 (1999) hepth/9905139.

60. J. Scherk and J. H. Schwarz, "How To Get Masses From Extra Dimensions," Nucl. Phys. B 153, 61 (1979).

61. I. Antoniadis and M. Quiros, "Supersymmetry breaking in M-theory and gaugino condensation," Nucl. Phys. B 505, 109 (1997) hep-th/9705037. 